\title{
Increased Morbidity and Mortality in COVID-19 Patients with Liver Injury
}

\author{
Mohammad Arsalan Siddiqui ${ }^{1}$ (1) - Suraj Suresh ${ }^{1}$. Stephen Simmer ${ }^{1} \cdot$ Mouhanna Abu-Ghanimeh $^{1} \cdot$ Megan Karrick $^{2}$. \\ Faisal Nimri ${ }^{2} \cdot$ Maher Musleh $^{2} \cdot$ Vivek Mediratta $^{1} \cdot$ Mustafa Al-Shammari $^{1} \cdot$ Sarah Russell $^{1}$ • Jessica Jou ${ }^{1}$. \\ Duyen Dang ${ }^{1} \cdot$ Reena Salgia $^{1} \cdot$ Tobias Zuchelli $^{1}$
}

Received: 24 October 2020 / Accepted: 14 April 2021 / Published online: 4 May 2021

(c) The Author(s), under exclusive licence to Springer Science+Business Media, LLC, part of Springer Nature 2021

\begin{abstract}
Background There is a high prevalence of liver injury (LI) in patients with coronavirus disease 2019 (COVID-19); however, few large-scale studies assessing risk factors and clinical outcomes in these patients have been done.

Aims To evaluate the risk factors and clinical outcomes associated with LI in a large inpatient cohort of COVID-19 patients. Methods Adult patients with COVID-19 between March 1 and April 30, 2020, were included. LI was defined as peak levels of alanine aminotransferase/aspartate aminotransferase that were 3 times the ULN or peak levels in alkaline phosphatase/ total bilirubin that were 2 times the ULN. Mild elevation in liver enzymes (MEL) was defined as abnormal peak liver enzyme levels lower than the threshold for LI. Patients with MEL and LI were compared to a control group comprising patients with normal liver enzymes throughout hospitalization.

Results Of 1935 hospitalized COVID-19 patients, 1031 (53.2\%) had MEL and 396 (20.5\%) had LI. Compared to control patients, MEL and LI groups contained proportionately more men. Patients in the MEL cohort were older compared to control, and African-Americans were more highly represented in the LI group. Patients with LI had an increased risk of mortality (relative risk [RR] 4.26), intensive care unit admission (RR, 5.52), intubation (RR, 11.01), 30-day readmission (RR, 1.81), length of hospitalization, and intensive care unit stay (10.49 and 10.06 days, respectively) compared to control. Conclusion Our study showed that patients with COVID-19 who presented with LI had a significantly increased risk of mortality and poor clinical outcomes.
\end{abstract}

Keywords COVID-19 $\cdot$ SARS-CoV-2 $\cdot$ Liver injury $\cdot$ Risk factors $\cdot$ Clinical outcomes

\section{Introduction}

Coronaviruses are a family of viruses that cause respiratory and intestinal illness in humans [1]. These viruses can affect the upper respiratory tract, manifesting as diseases like the common cold, or in more extreme cases, pneumonia [2]. Severe acute respiratory syndrome coronavirus-2 (SARS-CoV-2) is the virus that causes the coronavirus disease 2019 (COVID-19). The first cases of COVID-19

Mohammad Arsalan Siddiqui

msiddiq1@hfhs.org

1 Department of Gastroenterology and Hepatology, Henry Ford Hospital, 2799 West Grand Blvd., Detroit, MI 48202, USA

2 Department of Internal Medicine, Henry Ford Hospital, 2799 West Grand Blvd., Detroit, MI 48202, USA emerged in December 2019 in the city of Wuhan in the Chinese province of Hubei, where most patients presented with viral pneumonia. Since then, COVID-19 has spread globally and is recognized as a global pandemic by the World Health Organization [3].

Recent studies have shown that COVID-19 can affect multiple organs, and more than half of patients with COVID19 can have varying levels of liver enzyme abnormalities [4]. Some reports have shown that the liver is the most frequently involved organ outside of the respiratory system [5-7]. According to one study, the SARS-CoV-2 virus may bind to angiotensin-converting enzyme 2 (ACE2) on cholangiocytes, resulting in cholangiocyte dysfunction and hence inducing a systemic inflammatory response that can lead to liver injury (LI) [8].

Although there has been a fair amount of recent research on liver enzyme abnormalities and associated clinical 
characteristics among patients with COVID-19, most of these studies have been relatively small and were not conducted on a US population. Also, only a few studies focused specifically on hospital-based outcomes. In this study, our objective was to determine the risk factors and clinical outcomes associated with LI in a large cohort of patients who were hospitalized with COVID-19.

\section{Methods}

\section{Study Design}

This study was approved by the Henry Ford Health System Institutional Review Board. This study was a retrospective medical record review at a large tertiary care health system in Detroit, Michigan. Patients with SARS-CoV-2 infection confirmed by positive polymerase chain reaction testing of a nasopharyngeal specimen were included. The study cohort consisted of patients who were admitted and discharged to any of the five hospitals within the Henry Ford Health System between March 1 and April 30, 2020. Patients who were discharged directly from the emergency department or evaluated in outpatient clinics were not included. If patients were admitted multiple times during the study interval, only the index admission following a positive COVID-19 test result was considered.

\section{Data Collection}

A team of physicians performed a manual retrospective chart review on a standardized data collection form using the electronic medical record. Demographic data were collected including sex, age, race, body mass index, and current or prior cigarette use. The presence of baseline medical comorbidities including diabetes, sleep apnea, chronic obstructive pulmonary disease, asthma, hypertension, cardiovascular disease, and malignancy was recorded. Cardiovascular disease was defined by the presence of coronary artery disease, prior myocardial infarction, cerebrovascular disease, congestive heart failure, cardiac arrhythmia, or congenital heart disease.

Patients' peak liver enzymes during the index admission were also collected. Mild elevation in liver enzymes (MEL) was defined as peak levels of alanine aminotransferase, aspartate aminotransferase, alkaline phosphatase, and total bilirubin above the upper limit of normal (ULN) levels but lower than the threshold level for LI. LI was defined as peak levels of alanine aminotransferase/aspartate aminotransferase 3 times the ULN level or peak alkaline phosphatase/ total bilirubin levels 2 times the ULN level. ULN threshold values of alanine aminotransferase $52 \mathrm{IU} / \mathrm{L}$, aspartate aminotransferase $35 \mathrm{IU} / \mathrm{L}$, total bilirubin $1.2 \mathrm{mg} / \mathrm{dL}$, alkaline phosphatase $140 \mathrm{IU} / \mathrm{L}$ were used. These threshold values were defined by the health system's laboratory experts. Two cohorts, patients with MEL and patients with LI, were compared with a control group that comprised COVID-19 patients with normal LFTs at presentation and throughout hospitalization. Primary outcomes were 60-day mortality, intensive care unit (ICU) admission, intubation, 30-day readmission, length of hospitalization and length of ICU stay.

\section{Statistical Analysis}

All continuous data were reported as means and standard deviations, while categorical data were reported as counts and column percentages. For continuous variables, univariate two-group comparisons were performed using one-way analysis of variance tests if the variable was normally distributed and Kruskal-Wallis tests if the variable was not normally distributed. For categorical variables, univariate two-group comparisons were made using Chi-square tests when expected cell counts were $>5$ and Fisher's exact tests when expected cell counts were $<5$. Relative risk (RR) for the outcomes of interest was assessed using the log-binomial method. Pairwise post hoc tests were performed using a Bonferroni adjustment. To compare patients with cirrhosis, chronic liver disease, and those without chronic liver disease, generalized linear mixed modeling was used to account for the fact that these groups were not mutually exclusive. A Tukey-Kramer adjustment was used for multiple comparisons. For binary outcome variables, statistical significance was set at $P<0.05$. All analyses were performed using SAS 9.4 (SAS Institute Inc., Cary, NC).

\section{Results}

\section{Characteristics of Patients with COVID-19 with Elevated Liver Enzyme Levels}

A total of 1935 patients who tested positive for COVID-19 and were hospitalized between March 1 and April 30, 2020, were included in the study. Of the 1935 patients 972 were men $(50.2 \%)$ and 963 were women $(49.8 \%)$, with a mean (standard deviation) age of 61.7 (17) years. Five hundred and eight patients (26.2\%) with normal liver enzymes (control group), 1031 patients (53.2\%) with MEL, and 396 patients (20.5\%) with LI. Men were more common in the MEL group (50\%; $P=0.0006)$ and LI groups $(64 \% ; P<0.0001)$ compared to the control group of patients with normal liver enzymes (40\%) (Table 1).

Although patients in the MEL cohort were on average older compared to controls (65.1 vs. 61.7 years; $P=0.0010$ ), the difference in age between the LI cohort and control group was not significant ( 63.4 vs. 61.7 years; $P=0.4270$ ). 
Table 1 Comparison of patient clinical characteristics (MEL vs. control and LI vs. control) $(\mathrm{N}=1935)$

\begin{tabular}{|c|c|c|c|c|c|c|}
\hline Variable & ${ }^{\mathrm{a}}$ Control $(n=508)$ & $\operatorname{MEL}(n=1031)$ & LI $(n=396)$ & Overall ${ }^{\mathrm{b}} P$ value & $\begin{array}{l}\text { Control versus } \\
\text { MEL }^{\mathrm{b}} P \text { value }\end{array}$ & $\begin{array}{l}\text { Control versus } \\
\text { LI }^{\mathrm{b}} P \text { value }\end{array}$ \\
\hline Sex, $N(\%)$ & & & & $<0.0001$ & 0.0006 & $<0.0001$ \\
\hline Female & $304(60 \%)$ & $516(50 \%)$ & $143(36 \%)$ & & & \\
\hline Male & $204(40 \%)$ & $515(50 \%)$ & $253(64 \%)$ & & & \\
\hline Age, years, mean (SD) & $61.7(17.7)$ & $65.1(15.7)$ & $63.4(15.9)$ & 0.0006 & 0.0010 & 0.4270 \\
\hline Race/ethnicity, $N(\%)$ & & & & 0.0319 & 1.000 & 0.0260 \\
\hline Black & $278(56 \%)$ & $569(58 \%)$ & $238(64 \%)$ & & & \\
\hline White & $178(36 \%)$ & $341(35 \%)$ & $106(29 \%)$ & & & \\
\hline Asian & $8(2 \%)$ & $16(2 \%)$ & $15(4 \%)$ & & & \\
\hline Hispanic & $2(0 \%)$ & $4(0 \%)$ & $1(0 \%)$ & & & \\
\hline Other & $28(6 \%)$ & $50(5 \%)$ & $13(3 \%)$ & & & \\
\hline BMI, $\mathrm{kg} / \mathrm{m}^{2}$, mean $(\mathrm{SD})$ & $31.6(9.2)$ & $31.6(8.5)$ & $31.7(8.6)$ & 0.9796 & 1.000 & 1.000 \\
\hline \multicolumn{7}{|l|}{ Comorbidities, $N(\%)$} \\
\hline Diabetes & $206(41 \%)$ & $387(38 \%)$ & $153(39 \%)$ & 0.5202 & 0.5062 & 1.000 \\
\hline Sleep apnea & $53(10 \%)$ & $105(10 \%)$ & $35(9 \%)$ & 0.6896 & 1.000 & 0.8446 \\
\hline Hypertension & $333(66 \%)$ & $711(69 \%)$ & $271(68 \%)$ & 0.3926 & 0.3558 & 0.7220 \\
\hline Cardiovascular disease & $146(29 \%)$ & $288(28 \%)$ & $125(31 \%)$ & 0.4030 & 1.000 & 0.7152 \\
\hline Malignancy & $32(6 \%)$ & $73(7 \%)$ & $37(9 \%)$ & 0.1984 & 1.000 & 0.1744 \\
\hline Chronic liver disease & $14(3 \%)$ & $41(4 \%)$ & $11(3 \%)$ & 0.3443 & 0.4502 & 1.000 \\
\hline CRP mg/dL (peak), mean (SD) & $9.5(7.8)$ & $13.7(8.9)$ & $18.5(10.7)$ & $<0.0001$ & $<0.0001$ & $<0.0001$ \\
\hline Ferritin ng/mL (peak), mean (SD) & $586.1(782.3)$ & $1144.9(1855.9)$ & $3388.7(7501.1)$ & $<0.0001$ & 0.0352 & $<0.0001$ \\
\hline
\end{tabular}

BMI, body mass index; CRP, C-reactive protein; LI, liver injury; MEL, mild elevation in liver enzymes; SD, standard deviation

${ }^{a}$ Control patients had all liver enzymes within reference range

${ }^{\mathrm{b}} P$ values reported using one-way analysis of variance test for age and BMI and Chi-square test or Fisher's exact test for other patient characteristics

African-Americans were more highly represented in the LI group (58\%; $P=0.0260)$. Between all 3 groups, there was no difference in patient comorbidities including body mass index, diabetes, sleep apnea, hypertension, cardiovascular disease, and malignancy (Table 1).

\section{Laboratory Parameters of Patients with COVID-19 with Elevated Liver Enzyme Levels}

Among patients with LI, 241 (61\%) had a hepatocellular pattern, $20(5 \%)$ had a cholestatic pattern, and $135(34 \%)$ had a mixed pattern. Of the 1031 patients with MEL, the median alanine aminotransferase was 39 IU/L (range 5-156), the median aspartate aminotransferase was 52 IU/L (range 15-105), the median alkaline phosphatase was $71 \mathrm{IU} / \mathrm{L}$ (range 24-258), and the median total bilirubin was $0.8 \mathrm{mg} /$ $\mathrm{dL}$ (range 0.2-2.4). Of the 396 patients with LI, the median alanine aminotransferase was 131.5 IU/L (range 3-8518), the median aspartate aminotransferase was $141.5 \mathrm{IU} / \mathrm{L}$ (range 13-19,689), the median alkaline phosphatase was 97.5 IU/L (range 36-830), and the median total bilirubin was $1 \mathrm{mg} / \mathrm{dL}$ (range 0.2-22.7). Elevation in C-reactive protein and ferritin was correlated with severity of liver enzyme elevation. Mean C-reactive protein was $9.5 \mathrm{mg} / \mathrm{dL}$ in the control cohort, $13.7 \mathrm{mg} / \mathrm{dL}$ in the MEL cohort, and $18.5 \mathrm{mg} /$ $\mathrm{dL}$ in the LI cohort $(P<0.0001)$. Similarly, mean ferritin was $586 \mathrm{ng} / \mathrm{mL}$ in the control cohort, $1145 \mathrm{ng} / \mathrm{mL}$ in the MEL cohort, and $3389 \mathrm{ng} / \mathrm{mL}$ in the LI cohort (Table 1).

\section{Clinical Outcomes}

Patients with LI had an increased risk of mortality (RR, 4.26; 95\% CI 3.12-5.81; $P<0.0001$ ), ICU admission (RR, 5.52; 95\% CI 4.07- 7.49; $P<0.0001)$, intubation (RR, 11.01; 95\% CI 6.97-17.34; $P<0.0001)$, and 30-day readmission (RR, $1.81 ; 95 \%$ CI $1.17-2.80 ; P<0.0076)$ compared to the controls. Although patients with MEL had an increased risk of mortality (RR, 2.16; 95\% CI 1.59-2.95; $P<0.0001$ ), ICU admission (RR, 2.48; 95\% CI 1.82-3.37; $P<0.0001$ ), and intubation (RR,3.76; 95\% CI 2.36-5.99; $P<0.0001$ ) compared to the control group, there was no significant difference in the risk of 30-day readmission (RR, 1.25 ; $95 \%$ CI $0.86-1.82 ; P=0.2415$ ) (Table 2). Compared to the control group, patients with LI had a fourfold higher rate of mortality, fivefold higher rate of ICU admissions, 11-fold higher rate of intubation, and twofold higher rate of 30-day 
Table 2 Relative risks' main outcomes in patients with MEL or LI compared to control

\begin{tabular}{llllll}
\hline Outcome & \multicolumn{2}{l}{$\begin{array}{l}\text { Mildly elevated liver enzymes versus } \\
\text { control }\end{array}$} & & \multicolumn{2}{l}{ Liver injury versus control } \\
\cline { 2 - 2 } & Relative risk $(95 \% \mathrm{CI})$ & ${ }^{\mathrm{a}} P$ value & & Relative risk $(95 \% \mathrm{CI})$ & ${ }^{\mathrm{a}} P$ value \\
\hline 60-day mortality & $2.16(1.59-2.95)$ & $<0.0001$ & & $4.26(3.12-5.81)$ & $<0.0001$ \\
ICU admission & $2.5(1.8-3.4)$ & $<0.0001$ & & $5.52(4.07-7.49)$ & $<0.0001$ \\
Intubation & $3.76(2.36-5.99)$ & $<0.0001$ & & $11.01(6.97-17.34)$ & $<0.0001$ \\
30-day readmission & $1.25(0.86-1.82)$ & 0.2415 & & $1.81(1.17-2.80)$ & 0.0076 \\
\hline
\end{tabular}

ICU, intensive care unit; LI, liver injury; MEL, mild elevation in liver enzymes; RR, relative risk ${ }^{\text {a }} P$ values reported using Chi-square test readmissions (Fig. 1). Moreover, patients with LI and MEL had a longer length of hospitalization (10.5 days, $P<0.0001$ and 7.6 days, $P<0.0001)$ and ICU stay $(10.1$ days, $P<0.0001$ and 8.1 days, $P<0.0154)$ compared to control.

Among the patients in our study, 66 (3.4\%) had previously been diagnosed with chronic liver disease, 18 of whom had cirrhosis. The cause of the underlying liver disease was mainly related to Hepatitis C and NAFLD/NASH, which together comprised $73 \%$ of this cohort $(57.5 \%$ and $15 \%$, respectively). Fourteen of these patients did not have elevated liver enzymes during their hospitalization, two of whom were previously diagnosed with cirrhosis. Patients with cirrhosis had a higher mortality rate (RR, 2.19; $95 \%$ CI 1.33-3.62; $P=0.0022$ ) compared to controls, while patients with chronic liver disease without preexisting cirrhosis did not have a significantly different mortality rate (RR, 1.40; 95\% CI 0.93-2.09; $P=0.1062$ ). Patients with chronic liver disease both with and without preexisting cirrhosis showed no significant difference in ICU admissions, intubation, and 30-day readmissions compared to patients without chronic liver disease (Table 3 ).

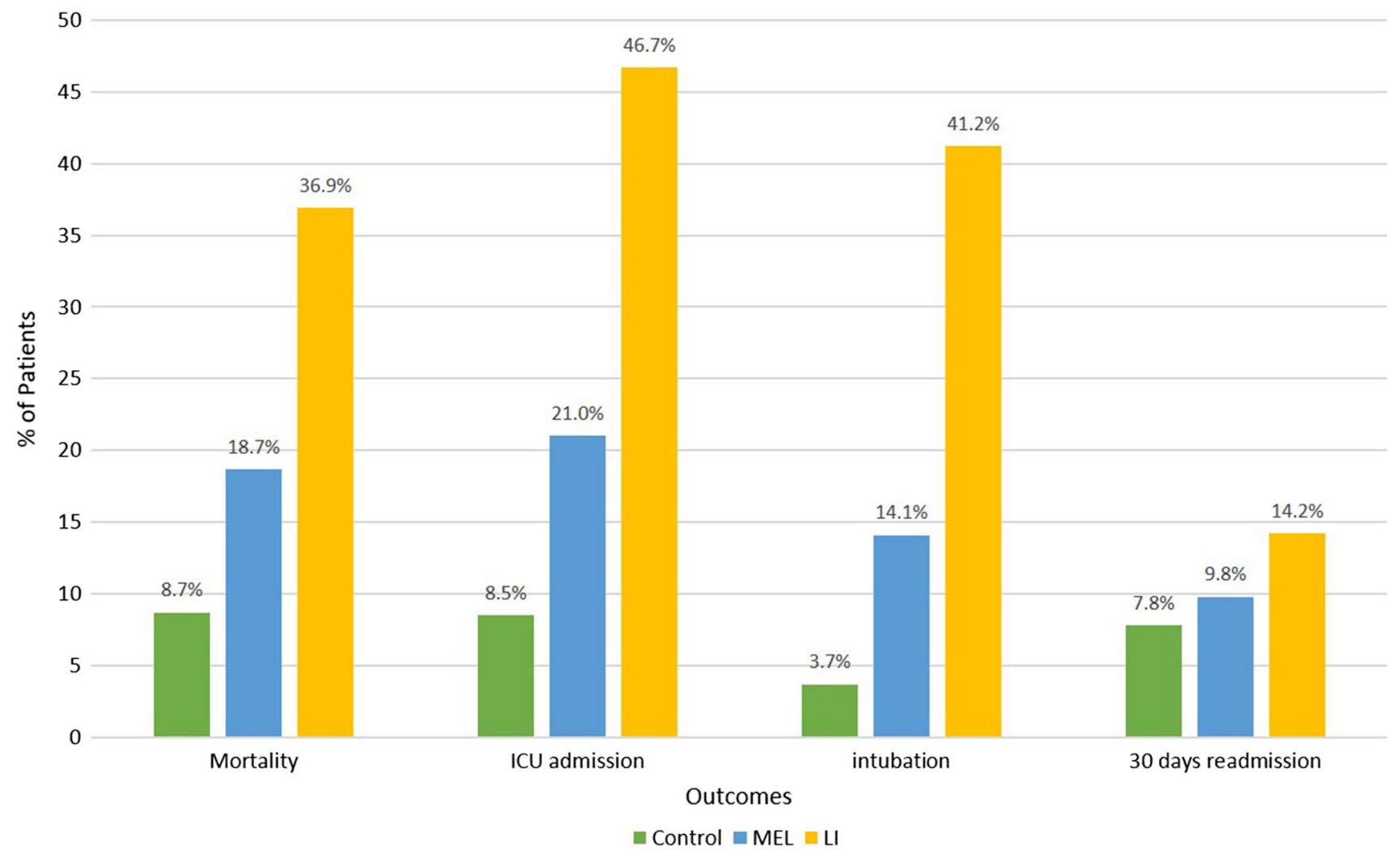

ICU, intensive care unit; LI, liver injury; MEL, mild elevation in liver enzymes.

Fig. 1 Incidence of adverse outcomes in patients with MEL and LI versus control. Abbreviations: ICU, intensive care unit; LI, liver injury; MEL, mild elevation in liver enzymes 
Table 3 Morbidity and mortality associated with cirrhosis and chronic liver disease without cirrhosis

\begin{tabular}{|c|c|c|c|c|}
\hline \multirow[t]{2}{*}{ Outcome } & \multicolumn{4}{|l|}{ Relative risk (95\% CI; ${ }^{\mathrm{a}} P$ value) } \\
\hline & Death & ICU admission & Intubation & 30-Day readmission \\
\hline Cirrhosis & $2.19(1.33-3.62 ; P=0.0022)$ & $0.41(0.11-1.55 ; P=0.1887)$ & $0.28(0.04-1.90 ; P=0.1922)$ & $2.35(0.86-6.42 ; P=0.0950)$ \\
\hline $\begin{array}{l}\text { Chronic liver } \\
\text { disease without } \\
\text { cirrhosis }\end{array}$ & $1.40(0.93-2.09 ; P=0.1062)$ & $1.06(0.69-1.63 ; P=0.7991)$ & $1.17(0.71-1.92 ; P=0.5323)$ & $1.24(0.58-2.67 ; P=0.5779)$ \\
\hline
\end{tabular}

ICU, intensive care unit

${ }^{\text {a }} P$ values reported using log-binomial method

\section{Discussion}

In this study, we observed that patients with MEL or LI had significantly higher intubation rates, ICU admission, mortality, length of hospitalization, and length of ICU stay compared to a control cohort of patients with normal liver enzymes. To our knowledge, this is the largest single-center retrospective study evaluating the relationship between liver enzyme elevation and COVID-19 severity in an inpatient cohort. Our study included patients who were hospitalized across multiple medical facilities within the larger Detroit metropolitan area, a region that was heavily affected by the COVID-19 pandemic. Our patient population was notably unique compared to other studies in that it had a significant proportion of African-Americans, who have been shown to be disproportionately affected by COVID-19 [9-11]. While African-Americans comprise $14 \%$ of the population in the state of Michigan, the city of Detroit has a large proportion of African-Americans, where $79 \%$ of citizens are black [12]. In our study, African-Americans made up 56\% of the study population and were also significantly more likely to have LI than patients of other races.

Our study aligns with current research that has shown that hepatic injury is common in COVID-19 infection and related to disease severity. Liver enzymes elevation was noted in $73.7 \%$ of our study group, where the rate of MEL was $53.2 \%$ and more severe LI was $20.5 \%$. Although the rate of liver enzyme elevation reported in our study was much higher than the $28.2 \%$ reported by Hao et al., it was more consistent with the $72.4 \%$ rate reported by Phipps et al. [13, 14]. Patients with MEL or LI had higher levels of systemic inflammation. The mean C-reactive protein in patients with MEL was $13.7 \mathrm{mg} / \mathrm{dL}$ and for patients with LI was $18.5 \mathrm{mg} /$ dL. Ferritin was also significantly higher with a mean of $1144 \mathrm{ng} / \mathrm{mL}$ in MEL and $3388 \mathrm{ng} / \mathrm{mL}$ in LI. The pattern of LI that we observed was similar to findings in other studies that reported elevated aspartate aminotransferase and alanine aminotransferase in severe disease [15]. Of the patients in our study, $61 \%$ developed hepatocellular injury, followed by a mixed pattern in $34 \%$ and a cholestatic pattern in only $5 \%$. Of unclear significance is the finding that male patients were more highly represented in both MEL and LI cohorts and that African-Americans were more likely to have LI than other races. Further studies will need to be done to corroborate these associations and find a causal relationship; however, the associations that we identified will help in risk stratification of a patient's disease severity. Other comorbidities including diabetes, sleep apnea, chronic obstructive pulmonary disease, asthma, hypertension, cardiovascular disease, and malignancy did not seem to be associated with these outcomes either.

The impact of chronic liver disease on the severity of COVID-19 was also evaluated in our study. The prevalence of underlying liver disease was $3.4 \%$, with almost a third of these patients having cirrhosis. Fourteen of those with chronic liver disease did not have elevation in their liver enzymes during their COVID-19 hospitalization. Two of these patients had underlying cirrhosis, and ten had HCV as their underlying disease process. The lack of elevations in these patients is curious, but can possibly be attributed to control of their underlying disease. Our study did not distinguish between HCV Ab-positive patients and NAT positive, and it is possible that those patients without elevations had been previously treated for their Hepatitis C. Further studies are required to delineate this relationship. Overall, our study showed that although cirrhosis conferred an increased risk of mortality related to controls, chronic liver disease without cirrhosis did not have an increased mortality rate and furthermore did not have higher rates of ICU admission, intubation, or 30-day readmission risk. These findings are in line with recent published data. The meta-analysis performed by Kovalic et al. found underlying chronic liver disease in 3\% of patients with COVID-19 infection [16]. These patients, which included those with cirrhosis, had an increased incidence of overall mortality as well as severe COVID-19 infection. Kim et al. performed a large US multicenter study looking specifically at patients with chronic liver disease and COVID-19 [17]. They showed an all-cause mortality of $14 \%$ with risk factors for increased mortality being alcohol-related liver disease, decompensated cirrhosis, and hepatocellular carcinoma. Our study population has some differences when compared to these studies; however, 
it reinforces the growing fund of knowledge that cirrhosis can confer an increased mortality risk and that this vulnerable population should take extra precaution to safeguard against contracting COVID-19.

The pathogenesis of LI in COVID-19 is still under investigation. However, it is thought to be related to one of three processes: direct viral infection of the liver, hepatic inflammation from activation of the innate immune system, or drug-induced LI. During the SARS-CoV epidemic that occurred in 2003 , it was noted that up to $66 \%$ of patients had elevated liver enzymes [18]. With that virus, which has $82 \%$ homology with SARS-CoV-2, there was evidence of direct LI with autopsy studies showing detectable virus in $41 \%$ of liver tissue and overexpression of a viral-encoded protein shown to induce apoptosis in liver cells [19-21]. In addition to significant genomic homology, the SARS-CoV-2 virus also has a similar mechanism of infection as its earlier counterpart, which is via the ACE2 receptor [18]. Although not highly expressed on hepatocytes, ACE2 has significant expression on cholangiocytes [8], and one role of these cells is an adaptive immune response mechanism in the liver, disruption of which could lead to hepatic damage [22]. Sterile inflammation has long been known to cause hepatic injury, as evidenced by disease processes such as autoimmune hepatitis and primary sclerosing cholangitis. The SARSCoV-2 virus has been showed to substantially elevate proinflammatory cytokines leading to a cytokine storm, manifested as elevated inflammatory markers with lymphopenia from reduction of T cells and NK cells [23]. Earlier studies have shown that these markers (e.g., elevated C-reactive protein $\geq 20 \mathrm{mg} / \mathrm{L}$ and lymphopenia $<1.1 \times 10^{\wedge} 9 / \mathrm{L}$ ) were independent risk factors for hepatic injury [24]. Finally, drug-induced LI is another likely etiology of some LI in COVID-19. The medication most implicated is lopinavir-ritonavir, where one case series reported that $55.4 \%$ of patients experienced LI after treatment [25]. So far, liver biopsy has not yielded a clear etiology. One reported biopsy from a COVID-19 patient autopsy showed nonspecific LI findings including moderate microvesicular steatosis and mild inflammation in the lobular portal region [26].

This study has several limitations. First, it may not be generalizable, since our data were collected from a single large health system, and the demographics of the patient cohort may not be reflective of the general population. Second, the resources available at a tertiary care facility such as ours are not readily available at many non-tertiary care centers, and this may have affected patient outcomes. Third, we only evaluated patients who required hospital admission and, therefore, were unable to capture the large proportion of patients who were cared for in the outpatient setting or were discharged home from the emergency department. Finally, as with any retrospective study, results may be subject to confounding bias from unknown variables. Two such variables are medication administration between the groups, which could have affected liver enzyme elevation, and lack of availability of baseline liver enzymes to serve as a reference point for MEL and LI, which again could confound the results. We recognize that using peak elevation of liver enzymes cannot indicate the timing of patient LI in relation to COVID-19 (i.e., whether the patient had an underlying liver condition, developed LI from drug toxicity, or developed LI as a sequela of viral infection). However, we propose that regardless of the timing or source of LI in COVID-19 patients, the presence of LI during a COVID-19 hospitalization may be indicative of the possibility of a severe outcome, highlighting LI as a bellwether of an unfavorable disease trajectory. Notably, we used similar ULN cutoffs for both men and women for parsimony, although a finer analysis would take gender-specific trends into consideration. Some of our ULN cutoffs may be different from those at other institutions, and this may have skewed our findings.

As of July 30, 2020, the World Health Organization reported over 16.8 million confirmed COVID-19 cases worldwide [27]. This global pandemic has caused significant morbidity and mortality, with over 662,095 deaths and untold economic damage. As of now, treatment is mainly supportive with consideration of anti-inflammatory agents, immunomodulatory therapies, certain antivirals, and antibody therapy such as convalescent plasma. Until a vaccine is developed and clinical trials on treatments yield more established and convincing treatment algorithms, prognostication of patients is critical.

\section{Conclusion}

In summary, we reported that patients with MEL or LI developed more severe disease and had increased intubation rates, ICU admission, mortality, length of hospitalization, and length of ICU stay as well increased 30-day readmission for patients with LI. This information is critical for optimal clinical decision making, and liver enzymes should be routinely monitored to allow for risk stratification and early treatment decisions. Further research looking at risk prediction models and pooling multicenter data should include LI as a key variable.

Acknowledgments We would like to thank Dr. Karla D. Passalacqua, Ph.D., at Henry Ford Hospital for editorial assistance, Abigail Chatfield for her help in statistical analysis, and Stephanie Stebens, MLIS, for her help in manuscript formatting.

Funding None.

\section{Declarations}

Conflicts of interest None. 
Ethical approval Approved by Institutional Review Board at Henry Ford Hospital.

Informed consent For this type of retrospective study, formal consent is not required.

\section{References}

1. Dong Y, Liang X, Yu X. Prognostic value of the dynamic changes in extra vascular lung water index and angiopoietin-2 in severe multiple trauma patients with acute respiratory distress syndrome [in Chinese]. Zhonghua Wei Zhong Bing Ji Jiu Yi Xиe 2019;31:571-576.

2. Niu P, Shen J, Zhu N, Lu R, Tan W. Two-tube multiplex real-time reverse transcription PCR to detect six human coronaviruses. Virol Sin. 2016;31:85-88.

3. World Health Organization. Clinical management of severe acute respiratory infection (SARI) when COVID-19 disease is suspected. Available at: https://apps.who.int/iris/bitstream/handle/ 10665/331446/WHO-2019-nCoV-clinical-2020.4-eng.pdf?seque nce $=1 \&$ isAllowed $=$ y. Accessed September 3, 2020.

4. Chau TN, Lee KC, Yao H et al. SARS-associated viral hepatitis caused by a novel coronavirus: report of three cases. Hepatology. 2004;39:302-310.

5. Hu LL, Wang WJ, Zhu QJ, Yang L. Novel coronavirus pneumonia related liver injury: etiological analysis and treatment strategy [in Chinese]. Zhonghua Gan Zang Bing Za Zhi 2020;28:97-99.

6. Zu ZY, Jiang MD, Xu PP et al. Coronavirus disease 2019 (COVID19): a perspective from China. Radiology. 2020;296:E15-E25.

7. Mao R, Liang J, Shen J et al. Implications of COVID-19 for patients with pre-existing digestive diseases. Lancet Gastroenterol Hepatol. 2020;5:425-427.

8. Chai X, Hu L, Zhang Y, et al. Specific ACE2 expression in cholangiocytes may cause liver damage after 2019-nCoV infection. bioRxiv. 2020;2020.02.03.931766.

9. Killerby ME, Link-Gelles R, Haight SC et al. Characteristics associated with hospitalization among patients with COVID-19 metropolitan Atlanta, Georgia, March-April 2020. MMWR Morb Mortal Wkly Rep. 2020;69:790-794.

10. Millett GA, Jones AT, Benkeser D et al. Assessing differential impacts of COVID-19 on black communities. Ann Epidemiol. 2020;47:37-44.

11. Price-Haywood EG, Burton J, Fort D, Seoane L. Hospitalization and mortality among black patients and white patients with Covid19. N Engl J Med. 2020;382:2534-2543.

12. United States Census Bureau. Quick Facts - Detroit City, Michigan. Available at: https://www.census.gov/quickfacts/detroitcit ymichigan. Accessed August 8, 2020.

13. Hao SR, Zhang SY, Lian JS et al. Liver enzyme elevation in coronavirus disease 2019: a multicenter, retrospective, cross-sectional study. Am J Gastroenterol. 2020;115:1075-1083.
14. Phipps MM, Barraza LH, LaSota ED et al. Acute liver injury in COVID-19: prevalence and association with clinical outcomes in a large US cohort. Hepatology. 2020;72:807-817.

15. Jiang $\mathrm{S}$, Wang $\mathrm{R}, \mathrm{Li} \mathrm{L}$ et al. Liver injury in critically ill and noncritically Ill COVID-19 patients: a multicenter, retrospective, observational study. Front Med (Lausanne). 2020;7:347.

16. Kovalic AJ, Satapathy SK, Thuluvath PJ. Prevalence of chronic liver disease in patients with COVID-19 and their clinical outcomes: a systematic review and meta-analysis. Hepatol Int. 2020;14:612-620.

17. Kim D, Adeniji N, Latt N, Kumar S et al. Predictors of outcomes of COVID-19 in patients with chronic liver disease: US multicenter study. Clin Gastroenterol Hepatol. 2020. https://doi.org/ 10.1016/j.cgh.2020.09.027.

18. Peiris JSM, Yuen KY, Osterhaus ADME, Stöhr K. The severe acute respiratory syndrome. $N$ Engl J Med. 2003;349:2431-2441.

19. Farcas GA, Poutanen SM, Mazzulli T et al. Fatal severe acute respiratory syndrome is associated with multiorgan involvement by coronavirus. J Infect Dis. 2005;191:193-197.

20. Tan YJ, Fielding BC, Goh PY et al. Overexpression of 7a, a protein specifically encoded by the severe acute respiratory syndrome coronavirus, induces apoptosis via a caspase-dependent pathway. J Virol. 2004;78:14043-14047.

21. Chan JFW, Kok KH, Zhu Z et al. Genomic characterization of the 2019 novel human-pathogenic coronavirus isolated from a patient with atypical pneumonia after visiting Wuhan. Emerg Microbes Infect. 2020;9:221-236 (Published correction appears in Emerg Microbes Infect. 2020;9:540).

22. Alqahtani SA, Schattenberg JM. Liver injury in COVID19: the current evidence. United European Gastroenterol J. 2020;8:509-519.

23. Cao X. COVID-19: immunopathology and its implications for therapy. Nat Rev Immunol. 2020;20:269-270.

24. Li L, Li S, Xu M, et al. Risk factors related to hepatic injury in patients with corona virus disease 2019. medRxiv. 2020;2020.02 .28.20028514.

25. Cai Q, Huang D, Ou P et al. COVID-19 in a designated infectious diseases hospital outside Hubei Province, China. Allergy 2020;75:1742-1752.

26. Xu Z, Shi L, Wang Y et al. Pathological findings of COVID-19 associated with acute respiratory distress syndrome. Lancet Respir Med. 2020;8:420-422 (Published correction appears in Lancet Respir Med. 2020;8:e26).

27. World Health Organization. Coronavirus disease (COVID-19) situation report - 192. Available at: https://www.who.int/docs/ default-source/coronaviruse/situation-reports/20200730-covid-19sitrep-192.pdf?sfvrsn=5e52901f_8. Accessed September 3, 2020.

Publisher's Note Springer Nature remains neutral with regard to jurisdictional claims in published maps and institutional affiliations. 